\title{
Cavity-Enhanced Photon Emission from a Single Germanium-Vacancy Center in a Diamond Membrane
}

Jensen, Rasmus Høy; Janitz, Erika; Fontana, Yannik Laurent; He, Yi; Gobron, Olivier; Radko, Ilya P.; Bhaskar, Mihir ; Evans, Ruffin; Rodríguez Rosenbluth, César Daniel; Childress, Lilian

Total number of authors:

12

Published in:

Physical Review Applied

Link to article, DOI:

10.1103/PhysRevApplied.13.064016

Publication date:

2020

Document Version

Publisher's PDF, also known as Version of record

Link back to DTU Orbit

Citation (APA):

Jensen, R. H., Janitz, E., Fontana, Y. L., He, Y., Gobron, O., Radko, I. P., Bhaskar, M., Evans, R., Rodríguez Rosenbluth, C. D., Childress, L., Huck, A., \& Andersen, U. L. (2020). Cavity-Enhanced Photon Emission from a Single Germanium-Vacancy Center in a Diamond Membrane. Physical Review Applied, 13, [064016]. https://doi.org/10.1103/PhysRevApplied.13.064016

\section{General rights}

Copyright and moral rights for the publications made accessible in the public portal are retained by the authors and/or other copyright owners and it is a condition of accessing publications that users recognise and abide by the legal requirements associated with these rights.

- Users may download and print one copy of any publication from the public portal for the purpose of private study or research.

- You may not further distribute the material or use it for any profit-making activity or commercial gain

- You may freely distribute the URL identifying the publication in the public portal 


\title{
Cavity-Enhanced Photon Emission from a Single Germanium-Vacancy Center in a Diamond Membrane
}

\author{
Rasmus Høy Jensen $\odot,{ }^{1},{ }^{*}, \dot{\dagger}$ Erika Janitz, $,{ }^{2, \dagger}, \dot{\dagger}$ Yannik Fontana, ${ }^{1}$ Yi He, ${ }^{3}$ Olivier Gobron, ${ }^{1}$ Ilya P. \\ Radko®, ${ }_{1}^{1}$ Mihir Bhaskar, ${ }^{4}$ Ruffin Evans, ${ }^{4}$ César Daniel Rodríguez Rosenblueth $\odot,{ }^{2}$ Lilian Childress $\odot,{ }^{2}$ \\ Alexander Huck, ${ }^{1}$ and Ulrik Lund Andersen ${ }^{1}$ \\ ${ }^{1}$ Center for Macroscopic Quantum States (bigQ), Department of Physics, Technical University of Denmark, \\ Lyngby, Denmark \\ ${ }^{2}$ Department of Physics, McGill University, Montreal, Quebec, Canada \\ ${ }^{3}$ Department of Electrical and Computer Engineering, Carnegie Mellon University, Pittsburgh, \\ Pennsylvania 15213, USA \\ ${ }^{4}$ Department of Physics, Harvard University, Cambridge, Massachusetts 02138, USA
}

(Received 18 December 2019; revised manuscript received 16 March 2020; accepted 30 March 2020; published 5 June 2020)

\begin{abstract}
The nitrogen-vacancy center in diamond has been explored extensively as a light-matter interface for quantum information applications, however, it is limited by low coherent photon emission and spectral instability. Here, we present a promising interface based on an alternative defect with superior optical properties (the germanium-vacancy) coupled to a finesse-11 000 fiber cavity, resulting in a $31_{-15}^{+11}$-fold increase in the spectral density of zero-phonon-line emission. This work sets the stage for cryogenic experiments, where we predict a measurable increase in the spontaneous emission rate.
\end{abstract}

DOI: 10.1103/PhysRevApplied.13.064016

\section{INTRODUCTION}

Defect centers in diamond can exhibit long-lived spin states that are accessible via coherent optical transitions [1-3], providing a promising platform for quantum nonlinear optics [4] or quantum networks [5,6]. There have been impressive steps towards creating such a network using the nitrogen-vacancy $(\mathrm{N}-V)$ center in diamond, with several critical components demonstrated in the last decade [7-13]. Nevertheless, optically mediated entanglement rates for $\mathrm{N}-V_{\mathrm{S}}$ have been limited to tens of $\mathrm{Hz}$ [14] due to the low fraction (3\%) of photons emitted into the coherent zero phonon line (ZPL), the difficulty of collecting these photons into a single optical mode, the long excited state lifetime (12 ns), and spectral diffusion of the emitter. This platform could be improved by coupling defects to an optical resonator, thereby increasing the ZPL emission rate into a well-defined mode. However, enhanced spectral diffusion near surfaces has thus far impeded attempts to realize stable N- $V$ centers in small-mode-volume optical cavities [15-17].

An alternative approach explores group-IV defect centers such as the silicon-vacancy (Si- $V$ ) $[18,19]$ and germanium-vacancy $(\mathrm{Ge}-V)$ centers $[20,21]$. While their

\footnotetext{
*rasjen@fysik.dtu.dk

†erika.janitz@mail.mcgill.ca

¥These authors contributed equally to this work.
}

spins interact strongly with phonons (necessitating low temperature [3] or high strain [22]), their optical properties are superior. These defects exhibit larger ZPL fractions $(60 \%-70 \%)$ [23], and strongly reduced spectral diffusion owing to their inversion symmetry [24-26]. Moreover, there is evidence that the Ge- $V$ also has high quantum efficiency [25]. Integrating Ge- $V \mathrm{~S}$ into optical resonators is therefore a promising step toward generating efficient or even deterministic spin-photon interactions [27].

Recently, two primary approaches for coupling diamond defects to optical cavities have emerged: nanophotonics and open-geometry microcavities. Emitters coupled to low-mode-volume nanophotonic resonators can exhibit high Purcell enhancement [15] and can even enter the highcooperativity regime $[27,28]$. Despite their inversion symmetry, $\mathrm{Si}-V_{\mathrm{S}}$ in such structures still suffer from increased spectral diffusion and inhomogeneous broadening due to nearby surfaces, lattice damage, and material strain [29]. This is further exacerbated for the Ge- $V$, since the heavier ion leads to increased implantation damage. Furthermore, the shorter ZPL wavelength $(603 \mathrm{~nm})$ makes fabrication more challenging, requiring smaller feature sizes with increased sensitivity to surface roughness.

In contrast to nanofabricated devices, open Fabry-Perot microcavities offer narrower resonator linewidths and in situ spectral and spatial tunability. Importantly, these systems accommodate microns-thick diamond membranes in which even $\mathrm{N}-V_{\mathrm{s}}$ can exhibit minimal spectral diffusion 
and bulklike optical properties [30]. Thus far, it has proven challenging to fabricate membranes with sufficient surface quality to achieve very high cavity finesse. This is particularly true when the optical energy is concentrated in the diamond (so-called "diamondlike" modes) due to a field antinode at the air-diamond interface [31]. Approaches using thinner (subwavelength) membranes may relax constraints on surface quality by precluding "diamondlike" mode confinement [32], but exposes defects to increased surface noise and material stress. Indeed, the only demonstration of open-cavity coupling of a single emitter in a diamond membrane used a finesse $\mathcal{F}=5260$ mode localized in air [17]. Already, this system achieved a 30-fold enhancement in N- $V$ center ZPL emission and a factor of 2 reduction in the excited-state lifetime, illustrating the potential of the open-cavity approach.

In this work, we observe coupling between a "diamondlike" mode of a Fabry-Perot microcavity and a single Ge- $V$ center in a membrane, thereby combining the advantageous optical properties of the Ge- $V$ with the flexibility of an open-geometry resonator. We build on previous roomtemperature experiments using nanodiamonds $[33,34]$ to observe funneling of emission into the cavity mode. Our experiment represents the first demonstration of single $\mathrm{Ge}-V$-cavity coupling and confirms that both the diamond membrane and cavity are of sufficient quality to support high-finesse $(\mathcal{F}>10000)$ "diamondlike" resonances. Furthermore, we observe and study the presence of a dark state in the Ge- $V$ level structure, elucidating new details regarding the emission dynamics. These results therefore represent an important step toward realizing an efficient spin-photon interface using diamond defects in open cavities.

\section{EXPERIMENTAL METHODS}

Our microcavities comprise a macroscopic flat mirror and a microscopic spherical mirror fabricated on the tip of a single-mode optical fiber (Thorlabs 630HP). The spherical dimple of the fiber mirror is machined using a laser ablation technique [35] with an effective radius of curvature $R=43.1 \pm 0.6 \mu \mathrm{m}$ [36]. Both substrates are coated with dielectric Bragg stack mirrors (LASEROPTIK); the flat mirror is low-index terminated such that the addition of the diamond membrane increases the mirror reflectivity, yielding an electric field antinode at the mirror-diamond interface. In contrast, the fiber mirror is high-index terminated with a field node at the air-mirror interface. The layers are numerically optimized to have matched transmissions $T_{\text {flat }}=T_{\text {fiber }}=70 \mathrm{ppm}$ at $603 \mathrm{~nm}$ and $T_{\text {flat }}=0.998$ and $T_{\text {fiber }}=0.007$ at the excitation wavelength $(532 \mathrm{~nm})$.

We incorporate emitters into the cavity by van der Waals bonding a diamond membrane containing Ge- $V \mathrm{~s}$ to the flat mirror [36], which is mounted on a three-axis stage with piezoelectric control (see Fig. 1). A long working

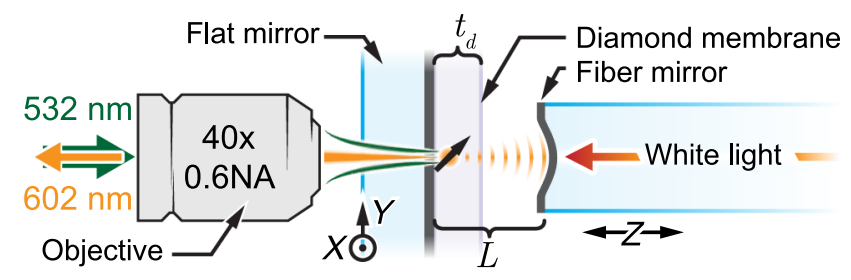

FIG. 1. Schematic of the cavity setup, where $L$ is the cavity length and $t_{d}=862_{-4}^{+1} \mathrm{~nm}$ is the membrane thickness.

distance objective (Olympus LUCPLFLN 40×, 0.6 NA) faces the backside of the flat mirror and is used for emitter excitation and cavity-mode collection; a separate stage facing the sample holds either an objective (Mitutoyo 100x Plan Apo, 0.7 NA) or the fiber mirror for confocal or cavity characterization, respectively. Furthermore, we use a piezoelectric scanner along the optical axis for fine tuning the cavity length and align the objectives and fiber mirror to the excitation laser, scanning the sample relative to the excitation spot to study different emitters.

\section{RESULTS}

We first characterize the membrane in the confocal configuration to map out the position of Ge- $V$ s. Confocal images are obtained by exciting emitters through the back of the flat mirror, while the emission is collected through the $100 \times$ objective and filtered with a passband of $600-605 \mathrm{~nm}$ before coupling into a single-mode fiber [Fig. 2(a)]. Here, bright spots correspond to single $\mathrm{Ge}-V \mathrm{~s}$ and contours are drawn at 4000 counts/s to qualitatively show emitter localization. We perform a detailed study of the well-isolated Ge- $V$ center marked with an arrow (henceforth referred to as the studied emitter). We confirmed that this was a single defect [measured intensity autocorrelation of $g^{2}(0)=0.25 \pm 0.16$ [36] ] with a lifetime of $\tau=6.0 \pm 0.1 \mathrm{~ns}$.

The modest fluorescence count rates observed can be attributed to a combination of low transmission in the collection path, narrow spectral filtering, and much of the emission either exiting through the flat mirror (which is designed for normal incidence) or being confined to the membrane in the form of guided modes [36]. Counts are further reduced by the presence of an additional dark state, which may be a different charge state of the Ge- $V$ center [37]. To quantify the impact of such a state, we probed the population dynamics of the studied emitter by measuring the second-order correlation function for varying excitation power [shown in Figs. 3(a) and 3(b) for different timescales]. We fit this data assuming a Poissonian background contribution parameterized by $\sigma=S /(S+B)$, where $S$ is the signal from the Ge- $V$ center and $B$ is background [38], resulting in

$$
g_{m}^{(2)}(\tau)=g^{(2)}(\tau) \sigma^{2}+1-\sigma^{2}
$$




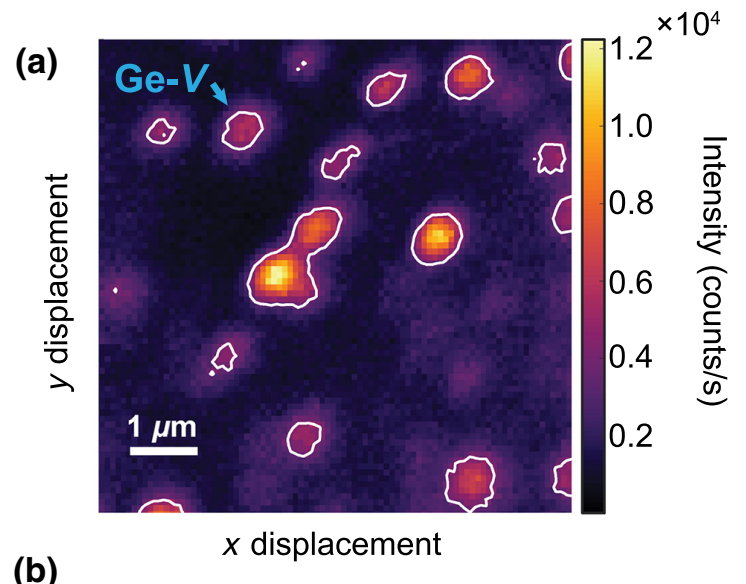

(b)

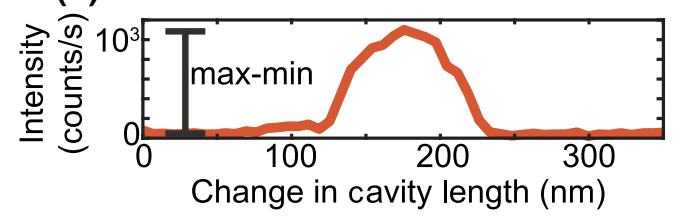

(c)
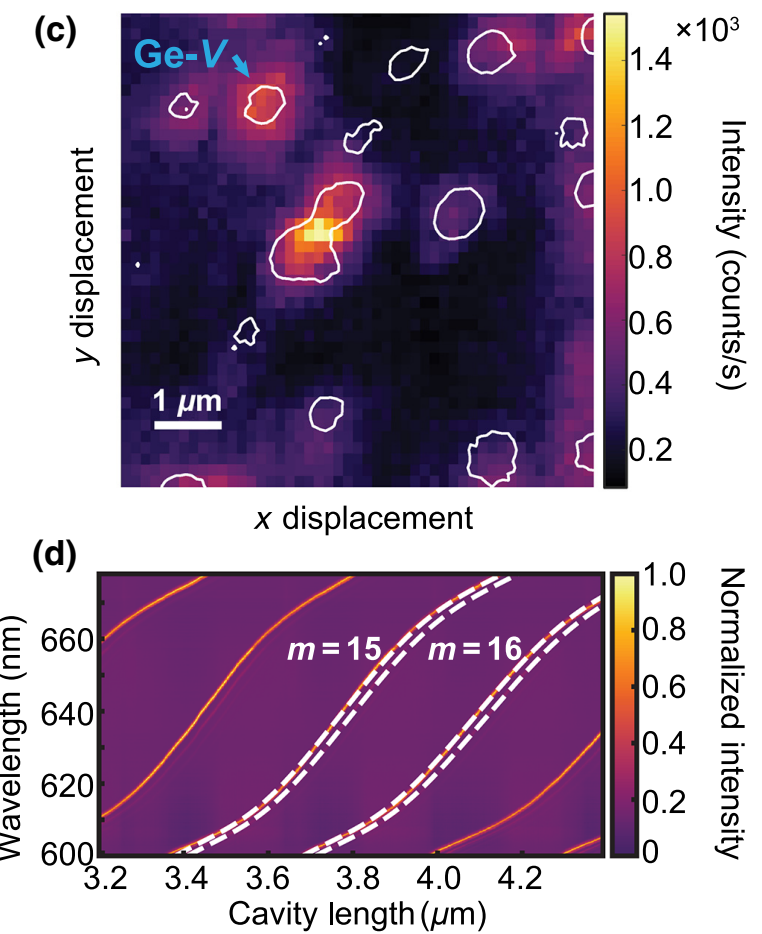

FIG. 2. (a) Confocal image of membrane fluorescence (600-605 nm, $P=19 \mathrm{~mW})$. White contours illustrate emitter localization and the studied $\mathrm{Ge}-V$ is indicated with an arrow. (b) Cavity-coupled emission as a function of resonator length for the studied $\operatorname{defect}(\mathcal{F}=11200 \pm 1700)$. The non-Lorentzian shape is determined by filters in the collection path. (c) Map of cavity-coupled emission amplitude ( $\max -\min )$ over the same area as in (a) $(P=19 \mathrm{~mW})$. Confocal contours are overlaid, and the studied Ge- $V$ indicated. (d) Broadband cavity transmission as a function of cavity length. Dashed lines correspond to numerical fits of the fundamental and firstorder transverse modes of the $m=15$ and 16 longitudinal modes.
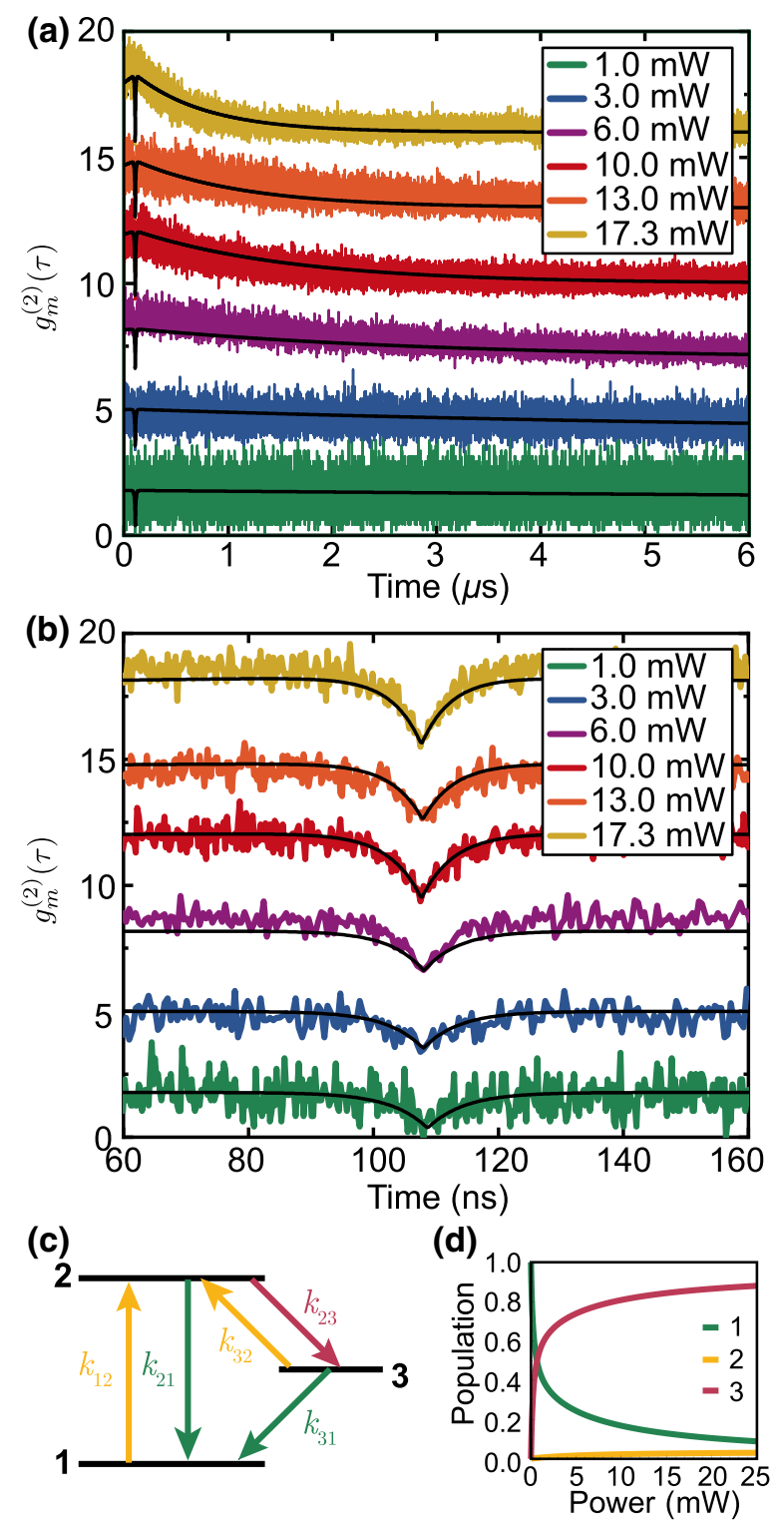

FIG. 3. (a) Power-dependent $g_{m}^{(2)}$ for the studied Ge- $V$ (subsequent measurements are offset by 3 for clarity). Fits to Eq. (1) are shown in black. (b) $g_{m}^{(2)}$ data and fits plotted at shorter timescales. (c) Equivalent three-level system for rate analysis. (d) State populations as a function of pump power.

where

$$
g^{(2)}(\tau)=1-(1+a) e^{-|\tau| / \tau_{1}}+a e^{-|\tau| / \tau_{2}} .
$$

The bunching of the $g_{m}^{(2)}$ function at high excitation powers implies that there are three or more states participating in the dynamics; we find that the system is well described by a three-level model including power-dependent shelving [Fig. 3(c), further details in Ref. [36] ] [34,39].

From this analysis, we calculate the populations as a function of excitation power [Fig. 3(d)]. We predict a 
dark-state equilibrium population of $96 \pm 18 \%$ at infinite 532-nm pump power, corresponding to a bright-state photon emission rate of $(6.8 \pm 0.9) \times 10^{6}$ photons/s. Reassuringly, there has been some evidence that the dark-state population can be reduced via laser repumping [28,37]. Moreover, nonradiative decay paths may further limit photon counts, as varying estimates of quantum efficiency have been reported recently [25,37,40,41]. Finally, photon emission is further limited by our inability to saturate most emitters due to the low absorption cross section at $532 \mathrm{~nm}$ [42].

We then replace the $100 \times$ objective with the fiber mirror and measure a cavity finesse of $\mathcal{F}=11200 \pm 1700$ at $603 \mathrm{~nm}$ [31]. Exciting the emitter with $532 \mathrm{~nm}$, we observe cavity-coupled emission as a function of cavity length [Fig. 2(b)]. The counts clearly peak when the cavity length is resonant with the thermally broadened ZPL; optical filtering results in a profile that deviates from the expected Lorentzian. We repeat this measurement at various positions on the membrane and plot the difference in measured counts in Fig. 2(c), where the lateral resolution is set by the excitation spot size $(1 \mu \mathrm{m})$ since the cavity waist diameter is larger $(3 \mu \mathrm{m})$. The contours obtained from the confocal measurement are overlaid for comparison, revealing a clear spatial correspondence between emitters in the confocal scan and high emission into the cavity. This measurement corresponds to emission into the $m=15$ longitudinal cavity mode, as determined by numerical fitting of the broadband cavity transmission measurements [Fig. 2(d)] [36].

Further evidence that we are probing a cavity-coupled Ge- $V$ can be obtained by comparing emitter spectra from both setups. Figure 4(a) shows a confocal spectrum for the studied emitter, which exhibits a strong ZPL emission around $603 \mathrm{~nm}$ with a FWHM linewidth of $\gamma^{*} /(2 \pi)=5.22 \pm 0.05 \mathrm{THz}$ [36]. A cavity spectrum can be obtained by exciting the emitter while scanning the cavity length and acquiring a spectrum at each position [Fig. 4(b)]. Integrating the emission along one mode using a 2-nm window about the resonance results in the light trace shown in Fig. 4(c), where the noise can be attributed to mechanical instability of the cavity; we also average the data using a 40-point window to show the underlying shape (dark trace). The peaks around 597 and $609 \mathrm{~nm}$ are due to mechanical disturbances causing the cavity to pass through the same resonance twice. The qualitative similarities between the confocal and cavity spectra further confirm that the collected emission is coming from a Ge- $V$.

\section{DISCUSSION}

Comparison of cavity-coupled and free-space fluorescence rates allows us to quantify cavity funneling, however we must carefully account for different excitation intensities arising from interference effects in the diamond membrane and cavity. We thus compare the

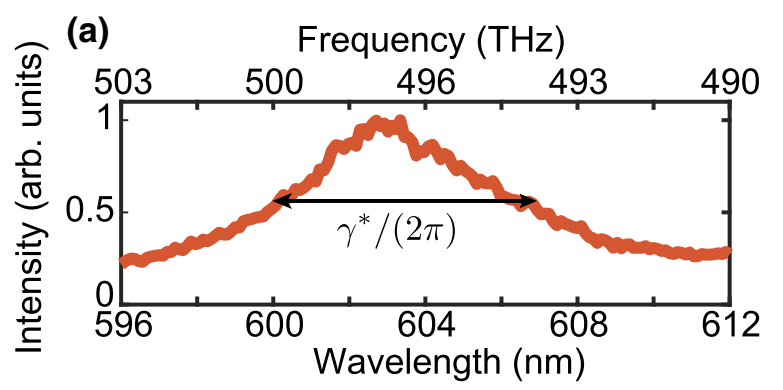

(b)
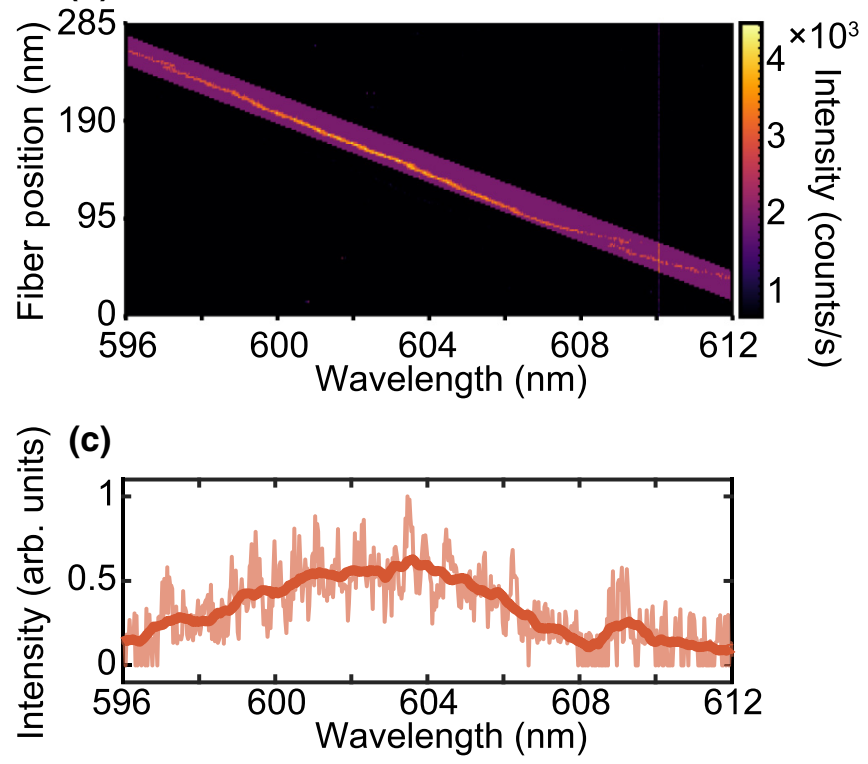

FIG. 4. (a) Ge- $V$ center spectrum taken in the confocal configuration. The pure dephasing rate $\gamma^{*} /(2 \pi)=5.22 \pm 0.05 \mathrm{THz}$ is indicated. (b) Ge- $V$ center fluorescence as a function of cavity length. The colored region represents the integration window. (c) Integrated spectrum taken in the cavity configuration. The light trace shows the raw data, and the dark trace has been averaged over 40 points.

saturating fluorescence counts at infinite pump power $\left(I^{\infty}\right)$ obtained by fitting saturation curves with the model $I(P)=I^{\infty}\left[P /\left(P+P_{\text {sat }}\right)\right]+c_{\text {bg }} P$, where $I$ is the observed count rate, $P$ is the excitation power, $P_{\text {sat }}$ is the saturation power, and $c_{\mathrm{bg}}$ accounts for a linear background. In the confocal setup, we measure a saturation power of $P_{\text {sat }}=3.9 \pm 0.3 \mathrm{~mW}$ and extract a saturating fluorescence count rate of $I_{\text {free,meas }}^{\infty}=4000 \pm 200$ counts $/ \mathrm{s}$. We can calculate the total emission rate of the defect using our estimate of the collection efficiency for the confocal path $\left[\eta_{\text {free }}=\left(3.5_{-1.5}^{+0.9}\right) \times 10^{-3}\right.$ counts $/$ photon $]$ as $I_{\text {free }}^{\infty}=I_{\text {free,meas }}^{\infty} / \eta_{\text {free }}=\left(1.2_{-0.3}^{+0.5}\right) \times 10^{6}$ photons $/ \mathrm{s} \quad$ [36]. Comparing this value to the maximum photon emission rate predicted at infinite pump power results in a quantum efficiency of $17_{-5}^{+8} \%$ for the studied Ge- $V$, which falls within the range of reported values $[25,37,40]$. Furthermore, we estimate the peak photon spectral density to be $90_{-20}^{+40}$ photons/(s GHz) [36]. 

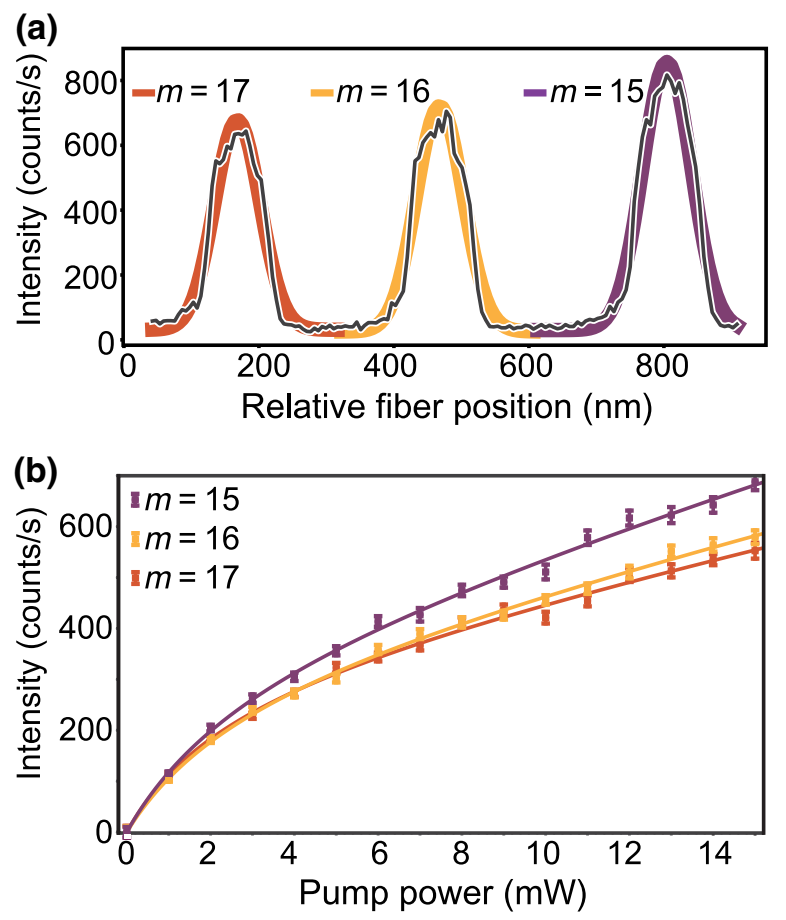

FIG. 5. (a) Longitudinal cavity scan $(P=14 \mathrm{~mW})$. The amplitudes of the Gaussian fits for $m=15,16$, and 17 are used to generate saturation curves. (b) Corresponding saturation curves, where error bars indicate one standard deviation on the fit amplitude.

We then study the saturation behavior of the emitter coupled to the $m=15,16$, and 17 longitudinal modes of the cavity. For each excitation power, we measure fluorescence as a function of cavity length [Fig. 5(a)]. The amplitudes of these resonances are estimated by fitting with Gaussians, and are plotted as a function of excitation power in Fig. 5(b). As expected, the cavitycoupled fluorescence decreases with mode number as the cavity mode volume increases. Fitting the $m=15$ data to the saturation model yields $P_{\text {sat }}=3.1 \pm 0.5 \mathrm{~mW}$ and $I_{\text {cav, } \text { meas }}^{\infty}=380 \pm 50$ counts $/ \mathrm{s}$. We estimate a collection efficiency of $\eta_{\text {cav }}=(8.2 \pm 1.2) \times 10^{-2}$ counts/photon for the cavity setup, resulting in a corrected saturating fluorescence count rate of $I_{\mathrm{cav}}^{\infty}=I_{\mathrm{cav}, \text { meas }}^{\infty} / \eta_{\mathrm{cav}}=$ $4700 \pm 900$ photons/s [36]. Moreover, we estimate a cavity linewidth of $\kappa /(2 \pi)=1.08 \pm 0.17 \mathrm{GHz}$, leading to a cavity-enhanced peak spectral density of $2800 \pm$ 700 photons / (s GHz), a factor of $31_{-15}^{+11}$ greater than what is obtained with the confocal measurements. The experimentally determined efficiency of emission into the $m=15$ mode is then $\beta_{\text {exp }}=I_{\text {cav }}^{\infty} /\left(I_{\text {free }}^{\infty}+I_{\text {cav }}^{\infty}\right)=0.40_{-0.19}^{+0.13} \%$ [36].

For comparison, we simulate $\beta$ for an emitter coupled to the $m=15$ cavity mode as a function of diamond thickness (Fig. 6) [36]. The solid line shows the result for the target implantation depth $(125 \mathrm{~nm})$ and the shaded regions reveal the spread for one and two times the standard deviation

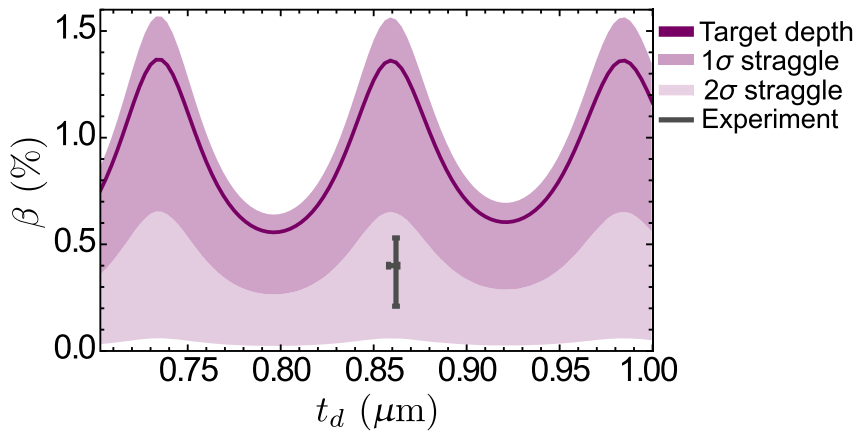

FIG. 6. Simulated $\beta$ for an emitter resonantly coupled to the $m=15$ mode as a function of diamond thickness, with the experimentally derived value of $\beta_{\exp }=0.40_{-0.19}^{+0.13} \%$ plotted at the extracted membrane thickness $t_{d}=862_{-4}^{+1} \mathrm{~nm}$.

in depth $(\sigma=20 \mathrm{~nm})$, resulting in $\beta$ between $0.06 \%$ and $1.55 \%$ at $t_{d}=862 \mathrm{~nm}$. The peaks in efficiency correspond to "diamondlike" modes, where the field in the diamond is maximized. We note that the observed $\beta_{\exp }$ lies at the lower range of simulated values, which we tentatively attribute to nonideal emitter location along the cavity axis from implantation straggle.

Cooling such a high-quality membrane to cryogenic temperatures should result in a narrowing of the ZPL, approaching the radiative limit $[\gamma /(2 \pi)=27 \mathrm{MHz}$ for $\tau=6.0 \mathrm{~ns}]$ and allowing for a Purcell enhancement of cavity-coupled optical transitions. The expected enhancement can be calculated from the extracted roomtemperature system parameters as $F_{p} \approx \frac{\gamma^{*}}{\xi \kappa} \beta_{\text {exp }}$, where $\xi=0.6$ is the Ge- $V$ Debye-Waller factor [43]. This would result in $F_{p}=32_{-16}^{+12}$ (assuming unity quantum efficiency and full depopulation of the dark state), corresponding to a $20_{-10^{+}}^{+7}$-times reduction in the excited state lifetime and $>95 \%$ of photons emitted into the ZPL. These values compare favorably with the current state of the art for open microcavities, where the ZPL of an $\mathrm{N}-V$ was enhanced by a comparable factor of $F_{p}=30$, with an excited-state lifetime reduction of only two due to the lower branching ratio [17].

\section{CONCLUSION}

In summary, we demonstrate coupling of a single Ge- $V$ center in a diamond membrane to a "diamondlike" mode of an open-cavity system, resulting in an $31_{-15}^{+11}$ times increase in the spectral density of single-photon emission. Moving forward, stable cryogenic operation at the ZPL frequency should be achievable using readily available cavity-locking techniques [44]; such resonant coupling would lead to a projected Purcell enhancement similar to what has been achieved for N- $V$ centers in open cavities [17] with a much larger reduction in the excited-state lifetime. This could be further improved by reducing the 
cavity mode volume via the mirror radius of curvature, where a shallow mirror with a $R \approx 5 \mu \mathrm{m}$ would increase the enhancement by a factor of 5 [45]. In parallel, the suitability of the Ge- $V$ as an efficient spin-photon interface should be confirmed through further characterization of the observed dark state and explicit measurement of the quantum efficiency in bulk diamond (which could be done using such a tuneable cavity). In the future, this platform could be easily adapted for coupling to emerging defect centers based on heavier group-IV elements such as the tin-vacancy $(\mathrm{Sn}-V)[46,47]$ and lead-vacancy $(\mathrm{Pb}-V)$ centers $[48,49]$, which should exhibit longer spin coherence times. This work therefore encompasses several steps toward realizing an efficient spin-photon interface for defect centers coupled to open microcavities.

\section{ACKNOWLEDGMENTS}

We thank Marie-Josée Gour for assistance with membrane fabrication. We acknowledge support from Canada Foundation for Innovation and Canada Research Chairs project 229003 and 231949, Fonds de Recherche Nature et Technologies FQRNT PR-253399, National Sciences and Engineering Research Council of Canada NSERC RGPIN 435554-13, l'Institut Transdisciplinaire d'Information Quantique (INTRIQ), the Danish Research Council through the Sapere Aude project DIMS, and the Danish National Research Foundation through the Center for Macroscopic Quantum States (bigQ, DNRF142). L. Childress is a CIFAR Fellow in the Quantum Information Science Program. Y. Fontana acknowledges support from SNSF Early Mobility Fellowship; CDRR is supported by a CONACYT fellowship; Y. Hi is supported by a MITACS Globalink internship.

[1] M. W. Doherty, N. B. Manson, P. Delaney, F. Jelezko, J. Wrachtrup, and L. C. Hollenberg, The nitrogen-vacancy colour centre in diamond, Phys. Rep. 528, 1 (2013).

[2] B. C. Rose, D. Huang, Z.-H. Zhang, P. Stevenson, A. M. Tyryshkin, S. Sangtawesin, S. Srinivasan, L. Loudin, M. L. Markham, A. M. Edmonds, et al., Observation of an environmentally insensitive solid-state spin defect in diamond, Science 361, 60 (2018).

[3] D. D. Sukachev, A. Sipahigil, C. T. Nguyen, M. K. Bhaskar, R. E. Evans, F. Jelezko, and M. D. Lukin, Silicon-Vacancy Spin Qubit in Diamond: A Quantum Memory Exceeding 10 ms with Single-Shot State Readout, Phys. Rev. Lett. 119, 223602 (2017).

[4] D. E. Chang, V. Vuletić, and M. D. Lukin, Quantum nonlinear optics - photon by photon, Nat. Photonics 8, 685 (2014).

[5] H. J. Kimble, The quantum internet, Nature 453, 1023 (2008).

[6] S. Wehner, D. Elkouss, and R. Hanson, Quantum internet: A vision for the road ahead, Science 362, eaam9288 (2018).
[7] E. Togan, Y. Chu, A. Trifonov, L. Jiang, J. Maze, L. Childress, M. G. Dutt, A. S. Sørensen, P. Hemmer, A. S. Zibrov, et al., Quantum entanglement between an optical photon and a solid-state spin qubit, Nature 466, 730 (2010).

[8] H. Bernien, L. Childress, L. Robledo, M. Markham, D. Twitchen, and R. Hanson, Two-Photon Quantum Interference from Separate Nitrogen Vacancy Centers in Diamond, Phys. Rev. Lett. 108, 043604 (2012).

[9] H. Bernien, B. Hensen, W. Pfaff, G. Koolstra, M. Blok, L. Robledo, T. Taminiau, M. Markham, D. Twitchen, L. Childress, et al., Heralded entanglement between solidstate qubits separated by three metres, Nature 497, 86 (2013).

[10] B. Hensen, H. Bernien, A. E. Dréau, A. Reiserer, N. Kalb, M. S. Blok, J. Ruitenberg, R. F. Vermeulen, R. N. Schouten, C. Abellán, et al., Loophole-free Bell inequality violation using electron spins separated by 1.3 kilometres, Nature 526, 682 (2015).

[11] W. Pfaff, B. Hensen, H. Bernien, S. B. van Dam, M. S. Blok, T. H. Taminiau, M. J. Tiggelman, R. N. Schouten, M. Markham, D. J. Twitchen, et al., Unconditional quantum teleportation between distant solid-state quantum bits, Science 345, 532 (2014).

[12] P. C. Maurer, G. Kucsko, C. Latta, L. Jiang, N. Y. Yao, S. D. Bennett, F. Pastawski, D. Hunger, N. Chisholm, M. Markham, et al., Room-temperature quantum bit memory exceeding one second, Science 336, 1283 (2012).

[13] N. Kalb, A. A. Reiserer, P. C. Humphreys, J. J. Bakermans, S. J. Kamerling, N. H. Nickerson, S. C. Benjamin, D. J. Twitchen, M. Markham, and R. Hanson, Entanglement distillation between solid-state quantum network nodes, Science 356, 928 (2017).

[14] P. C. Humphreys, N. Kalb, J. P. Morits, R. N. Schouten, R. F. Vermeulen, D. J. Twitchen, M. Markham, and R. Hanson, Deterministic delivery of remote entanglement on a quantum network, Nature 558, 268 (2018).

[15] A. Faraon, C. Santori, Z. Huang, V. M. Acosta, and R. G. Beausoleil, Coupling of Nitrogen-Vacancy Centers to Photonic Crystal Cavities in Monocrystalline Diamond, Phys. Rev. Lett. 109, 033604 (2012).

[16] L. Li, T. Schröder, E. H. Chen, M. Walsh, I. Bayn, J. Goldstein, O. Gaathon, M. E. Trusheim, M. Lu, J. Mower,et al., Coherent spin control of a nanocavity-enhanced qubit in diamond, Nat. Commun. 6, 6173 (2015).

[17] D. Riedel, I. Söllner, B. J. Shields, S. Starosielec, P. Appel, E. Neu, P. Maletinsky, and R. J. Warburton, Deterministic Enhancement of Coherent Photon Generation from a Nitrogen-Vacancy Center in Ultrapure Diamond, Phys. Rev. X 7, 031040 (2017).

[18] C. Hepp, T. Müller, V. Waselowski, J. N. Becker, B. Pingault, H. Sternschulte, D. Steinmüller-Nethl, A. Gali, J. R. Maze, M. Atatüre, et al., Electronic Structure of the Silicon Vacancy Color Center in Diamond, Phys. Rev. Lett. 112, 036405 (2014).

[19] L. J. Rogers, K. D. Jahnke, M. W. Doherty, A. Dietrich, L. P. McGuinness, C. Müller, T. Teraji, H. Sumiya, J. Isoya, N. B. Manson, et al., Electronic structure of the negatively charged silicon-vacancy center in diamond, Phys. Rev. B 89, 235101 (2014).

[20] P. Siyushev, M. H. Metsch, A. Ijaz, J. M. Binder, M. K. Bhaskar, D. D. Sukachev, A. Sipahigil, R. E. Evans, C. T. 
Nguyen, M. D. Lukin, et al., Optical and microwave control of germanium-vacancy center spins in diamond, Phys. Rev. B 96, 081201 (2017).

[21] T. Iwasaki, F. Ishibashi, Y. Miyamoto, Y. Doi, S. Kobayashi, T. Miyazaki, K. Tahara, K. D. Jahnke, L. J. Rogers, B. Naydenov, et al., Germanium-vacancy single color centers in diamond, Sci. Rep. 5, 12882 (2015).

[22] Y.-I. Sohn, S. Meesala, B. Pingault, H. A. Atikian, J. Holzgrafe, M. Gündoğan, C. Stavrakas, M. J. Stanley, A. Sipahigil, J. Choi, et al., Controlling the coherence of a diamond spin qubit through its strain environment, Nat. Commun. 9, 2012 (2018).

[23] G. M. H. Thiering and A. Gali, Ab Initio Magneto-Optical Spectrum of Group-IV Vacancy Color Centers in Diamond, Phys. Rev. X 8, 021063 (2018).

[24] R. E. Evans, A. Sipahigil, D. D. Sukachev, A. S. Zibrov, and M. D. Lukin, Narrow-Linewidth Homogeneous Optical Emitters in Diamond Nanostructures via Silicon Ion Implantation, Phys. Rev. Appl. 5, 044010 (2016).

[25] M. K. Bhaskar, D. D. Sukachev, A. Sipahigil, R. E. Evans, M. J. Burek, C. T. Nguyen, L. J. Rogers, P. Siyushev, M. H. Metsch, H. Park, et al., Quantum Nonlinear Optics with a Germanium-Vacancy Color Center in a Nanoscale Diamond Waveguide, Phys. Rev. Lett. 118, 223603 (2017).

[26] N. H. Wan, T.-J. Lu, K. C. Chen, M. P. Walsh, M. E. Trusheim, L. De Santis, E. A. Bersin, I. B. Harris, S. L. Mouradian, I. R. Christen, et al., Large-scale integration of near-indistinguishable artificial atoms in hybrid photonic circuits, arXiv:1911.05265 (2019).

[27] R. E. Evans, M. K. Bhaskar, D. D. Sukachev, C. T. Nguyen, A. Sipahigil, M. J. Burek, B. Machielse, G. H. Zhang, A. S. Zibrov, E. Bielejec, et al., Photon-mediated interactions between quantum emitters in a diamond nanocavity, Science 362, 662 (2018).

[28] A. Sipahigil, R. E. Evans, D. D. Sukachev, M. J. Burek, J. Borregaard, M. K. Bhaskar, C. T. Nguyen, J. L. Pacheco, H. A. Atikian, C. Meuwly, et al., An integrated diamond nanophotonics platform for quantum-optical networks, Science 354, 847 (2016).

[29] C. Nguyen, D. Sukachev, M. Bhaskar, B. Machielse, D. Levonian, E. Knall, P. Stroganov, C. Chia, M. Burek, R. Riedinger, et al., An integrated nanophotonic quantum register based on silicon-vacancy spins in diamond, Phys. Rev. B 100, 165428 (2019).

[30] M. Ruf, M. IJspeert, S. van Dam, N. de Jong, H. van den Berg, G. Evers, and R. Hanson, Optically coherent nitrogen-vacancy centers in micrometer-thin etched diamond membranes, Nano Lett. 19, 3987 (2019).

[31] E. Janitz, M. Ruf, M. Dimock, A. Bourassa, J. Sankey, and L. Childress, Fabry-Perot microcavity for diamond-based photonics, Phys. Rev. A 92, 043844 (2015).

[32] S. Häußler, J. Benedikter, K. Bray, B. Regan, A. Dietrich, J. Twamley, I. Aharonovich, D. Hunger, and A. Kubanek, Diamond photonics platform based on silicon vacancy centers in a single-crystal diamond membrane and a fiber cavity, Phys. Rev. B 99, 165310 (2019).

[33] R. Albrecht, A. Bommer, C. Deutsch, J. Reichel, and C. Becher, Coupling of a Single Nitrogen-Vacancy Center in Diamond to a Fiber-Based Microcavity, Phys. Rev. Lett. 110, 243602 (2013).
[34] J. Benedikter, H. Kaupp, T. Hümmer, Y. Liang, A. Bommer, C. Becher, A. Krueger, J. M. Smith, T. W. Hänsch, and D. Hunger, Cavity-Enhanced Single-Photon Source Based on the Silicon-Vacancy Center in Diamond, Phys. Rev. Appl. 7, 024031 (2017).

[35] D. Hunger, T. Steinmetz, Y. Colombe, C. Deutsch, T. W. Hänsch, and J. Reichel, A fiber Fabry-Perot cavity with high finesse, New J. Phys. 12, 065038 (2010).

[36] See Supplemental Material at http://link.aps.org/supple mental/10.1103/PhysRevApplied.13.064016 for further information regarding membrane fabrication, experimental characterization, and theoretical details. The Supplemental Material includes Refs. [50-62].

[37] D. Chen, Z. Mu, Y. Zhou, J. E. Fröch, A. Rasmit, C. Diederichs, N. Zheludev, I. Aharonovich, and W.-B. Gao, Optical Gating of Resonance Fluorescence from a Single Germanium Vacancy Color Center in Diamond, Phys. Rev. Lett. 123, 033602 (2019).

[38] R. Brouri, A. Beveratos, J.-P. Poizat, and P. Grangier, Photon antibunching in the fluorescence of individual color centers in diamond, Opt. Lett. 25, 1294 (2000).

[39] E. Neu, M. Agio, and C. Becher, Photophysics of single silicon vacancy centers in diamond: Implications for single photon emission, Opt. Express 20, 19956 (2012).

[40] K. Boldyrev, B. Mavrin, P. S. Sherin, and M. Popova, Bright luminescence of diamonds with $\mathrm{Ge}-\mathrm{V}$ centers, J. Lumin. 193, 119 (2018).

[41] M. Nguyen, N. Nikolay, C. Bradac, M. Kianinia, E. A. Ekimov, N. Mendelson, O. Benson, and I. Aharonovich, Photodynamics and quantum efficiency of germanium vacancy color centers in diamond, Adv. Photonics 1, 1 (2019).

[42] S. Häußler, G. Thiering, A. Dietrich, N. Waasem, T. Teraji, J. Isoya, T. Iwasaki, M. Hatano, F. Jelezko, A. Gali, et al., Photoluminescence excitation spectroscopy of $\mathrm{SiV}^{-}$and $\mathrm{GeV}^{-}$color center in diamond, New J. Phys. 19, 063036 (2017).

[43] Y. N. Palyanov, I. N. Kupriyanov, Y. M. Borzdov, and N. V. Surovtsev, Germanium: A new catalyst for diamond synthesis and a new optically active impurity in diamond, Sci. Rep. 5, 14789 (2015).

[44] E. Janitz, M. Ruf, Y. Fontana, J. Sankey, and L. Childress, High mechanical bandwidth fiber-coupled Fabry-Perot cavity, Opt. Express 25, 20932 (2017).

[45] D. Najer, M. Renggli, D. Riedel, S. Starosielec, and R. J. Warburton, Fabrication of mirror templates in silica with micron-sized radii of curvature, Appl. Phys. Lett. 110, 011101 (2017).

[46] M. E. Trusheim, B. Pingault, N. H. Wan, L. De Santis, K. C. Chen, M. Walsh, J. J. Rose, J. N. Becker, E. Bersin, G. Malladi, et al., Transform-Limited Photons From a Coherent Tin-Vacancy Spin in Diamond, Phys. Rev. Lett. 124, 023602 (2020).

[47] A. E. Rugar, C. Dory, S. Sun, and J. Vučković, Characterization of optical and spin properties of single tin-vacancy centers in diamond nanopillars, Phys. Rev. B 99, 205417 (2019).

[48] M. E. Trusheim, N. H. Wan, K. C. Chen, C. J. Ciccarino, J. Flick, R. Sundararaman, G. Malladi, E. Bersin, M. Walsh, B. Lienhard, et al., Lead-related quantum emitters in diamond, Phys. Rev. B 99, 075430 (2019). 
[49] S. D. Tchernij, T. Lühmann, T. Herzig, J. Küpper, A. Damin, S. Santonocito, M. Signorile, P. Traina, E. Moreva, F. Celegato, et al., Single-photon emitters in lead-implanted single-crystal diamond, ACS Photonics 5, 4864 (2018).

[50] B. J. M. Hausmann, B. Shields, Q. Quan, P. Maletinsky, M. McCutcheon, J. T. Choy, T. M. Babinec, A. Kubanek, A. Yacoby, M. D. Lukin, et al., Integrated diamond networks for quantum nanophotonics, Nano Lett. 12, 1578 (2012).

[51] P. Latawiec, V. Venkataraman, M. J. Burek, B. J. Hausmann, I. Bulu, and M. Lončar, On-chip diamond Raman laser, Optica 2, 924 (2015).

[52] P. Appel, E. Neu, M. Ganzhorn, A. Barfuss, M. Batzer, M. Gratz, A. Tschöpe, and P. Maletinsky, Fabrication of all diamond scanning probes for nanoscale magnetometry, Rev. Sci. Instrum. 87, 063703 (2016).

[53] J. F. Ziegler, M. D. Ziegler, and J. P. Biersack, SRIM -the stopping and range of ions in matter (2010), Nucl. Instrum. Methods Phys. Res., Sect. B 268, 1818 (2010).

[54] Y. Chu, N. de Leon, B. Shields, B. Hausmann, R. Evans, E. Togan, M. J. Burek, M. Markham, A. Stacey, A. Zibrov, et al., Coherent optical transitions in implanted nitrogen vacancy centers, Nano Lett. 14, 1982 (2014).
[55] W. Lukosz and R. E. Kunz, Light emission by magnetic and electric dipoles close to a plane interface. I. Total radiated power, J. Opt. Soc. Am. 67, 1607 (1977).

[56] K. A. Neyts, Simulation of light emission from thin-film microcavities, J. Opt. Soc. Am. A 15, 962 (1998).

[57] L. Polereckỳ, J. Hamrle, and B. D. MacCraith, Theory of the radiation of dipoles placed within a multilayer system, Appl. Opt. 39, 3968 (2000).

[58] C. Reed, J. Giergiel, J. Hemminger, and S. Ushioda, Dipole radiation in a multilayer geometry, Phys. Rev. B 36, 4990 (1987).

[59] C. C. Katsidis and D. I. Siapkas, General transfer-matrix method for optical multilayer systems with coherent, partially coherent, and incoherent interference, Appl. Opt. 41, 3978 (2002).

[60] O. Arnon, Loss mechanisms in dielectric optical interference devices, Appl. Opt. 16, 2147 (1977).

[61] S. B. van Dam, M. Ruf, and R. Hanson, Optimal design of diamond-air microcavities for quantum networks using an analytical approach, New J. Phys. 20, 115004 (2018).

[62] S. A. Solin and A. K. Ramdas, Raman spectrum of diamond, Phys. Rev. B 1, 1687 (1970). 